\title{
Editorial
}

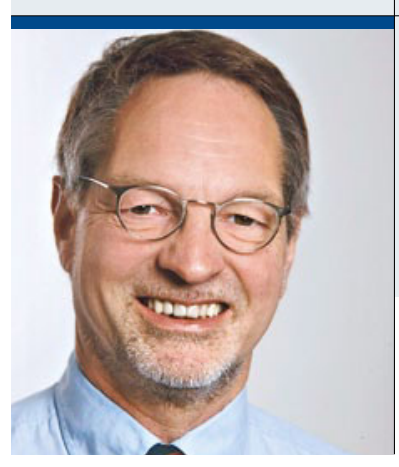

„Eine Umsetzung unserer urologischen Leitlinien

am Patienten wird letztlich nur über eine konsequente

Lobbyarbeit möglich sein."

Dr. med. Walther Grohmann,

Chefredakteur und niedergelassener Urologe, München

\section{Urologen in die Lobby!}

D

GU-Kongress im September: Ein Kollege spricht mich an: „Wir machen hier Wissenschaft und beste Leitlinien, aber in meiner Praxis finanziere ich bei einem Regelleistungsvolumen von weit unter 20 Euro alle Kassenpatienten durch meine Privatpraxis - mit Ach und Krach. Die Rettung des Euro ist „in“, der Staat wird wohl die Banken finanzieren, aber unsere Probleme und die Gesundheitsreform werden derzeit in der Öffentlichkeit totgeschwiegen. Nun soll die Gebührenordnung der Privaten Krankenversicherung reformiert werden. Vor den nächsten Bundestagswahlen will sich wohl niemand bei den regierenden Parteien daran die Finger verbrennen und wenn danach Herr Lauterbach das Sagen hat, können wir wohl alle einpacken. Wir haben halt keine Lobby, die wir jetzt so dringend bräuchten."

\section{Höherer Mitgliederbeitrag für mehr Professionalität}

Eine Stunde später: Bei der Vollversammlung des Berufsverbandes der Urologen ist der Saal bis auf den letzten Platz gefüllt. Auf der Tagesordnung steht unter anderem die Erhöhung der Mitgliedsbeiträge. Richard Berges, Urologe der Pan-Klinik Köln und Mitglied im URO-NEWS-Beirat, hält ein Plädoyer für diese Erhöhung vor dem Hintergrund einer Investition in die Zukunft. Professionalisierung und Lobbyarbeit im Berufsverband seien unumgänglich. Auch er spricht die nächsten Bundestagswahlen an und meint sinngemäß zur Illustration: Der derzeitige Gesundheitsminister habe vielleicht nach der kommenden Wahl genug Zeit, um gegen entsprechendes Honorar bei Bedarf mit ins Boot geholt zu werden. Die Rede zündet und mit überwältigender Mehrheit wird die Erhöhung der Mitgliedsbeiträge beschlossen.

\section{Urologen nicht gelistet}

Ein Monat nach dem DGU-Kongress: Dirk M. Potempa, niedergelassener Urologe in Garmisch und BDU-Landesvorsitzender, recherchiert in der Lobbyistenliste des deutschen Bundestags. Neben dem Deutschen Facharztverband, der sich hiermit sozusagen als Vertreter der Fachärzte beziehungsweise der 14 Berufsverbände präsentiert, sind dort nicht nur die Hämato-Onkologen mit ihrer Deutschen Gesellschaft und ihrem Berufsverband vertreten (www.bundestag.de/dokumente/parlamentsarchiv/ sachgeb/lobbyliste/lobbylisteaktuell.pdf). Auch die Pathologen, Laborärtze, die „Psycho“-Sparte, Kinderärzte, Internisten, Gynäkologen, Orthopäden, und Chirurgen geben sich die Klinke in die Hand. Somit sind eigentlich alle außer den urologischen Verbänden in der Liste angeführt! Es ist naheliegend, dass sich Politiker bei Rückfragen der Lobbyliste bedienen. Dirk Potempa hat zwischenzeitlich diesbezüglich mit dem BDU-Präsidenten Axel Schroeder Kontakt aufgenommen.

Fazit: Wir Urologen brauchen ein professionelle Interessenvertretung, besser schon heute als morgen. In Hinblick darauf sind die urologischen Leitlinien unter anderem eine wichtige Voraussetzung für die Rechtfertigung unserer Tätigkeit. Aber eine Umsetzung unserer urologischen Leitlinien am Patienten wird letztlich nur über eine konsequente Lobbyarbeit möglich sein. Ansonsten ist der - leitliniengerechte - Untergang der niedergelassenen Urologie absehbar.

Ihr

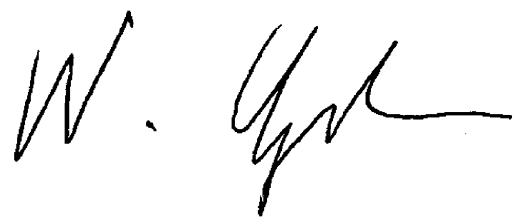

